Gynäkologische Endokrinologie 2015 .

13:64-68

DOI 10.1007/s10304-014-0660-0

Online publiziert: 16. November 2014

๑) Springer-Verlag Berlin Heidelberg 2014

\section{Redaktion}

L. Kiesel, Münster

A.O. Mueck, Tübingen

P. Stute, Bern

V. Widmer · P. Stute

Abteilung für Gynäkologische Endokrinologie und Reproduktionsmedizin, Frauenklinik Inselspital, Bern

\title{
Postmenopausale Hormontherapie und Kognition
}

\section{Methoden}

ist mit verschiedenen typischen Symptomen assoziiert. Unter anderem klagen über $60 \%$ der Frauen in den Wechseljahren über Gedächtniseinbußen [7]. Damit scheinen auch nicht sexualspezifische Wirkungen der Östrogene betroffen zu sein, z. B. die Einwirkung auf bestimmte Hirnregionen. Entsprechend liegt die Annahme nahe, dass kognitive Beschwerden ebenso wie vasomotorische Störungen durch exogene Östrogene reduziert werden können. Laborexperimente zeigen, dass Östrogene die Funktionen des Gehirns beeinflussen und eine neuroprotektive Wirkung haben [9].

\section{) In Laborexperimenten zeigen Östrogene eine neuroprotektive Wirkung}

In verschiedenen Beobachtungsstudien konnte ein positiver Effekt der postmenopausalen Hormontherapie (HT) auf die Kognition nachgewiesen werden [2]. Jedoch gibt es verschiedene klinische Studien, die diese Hypothese nicht unterstützen oder sogar widerlegen [6]. Die methodologischen Bedingungen der Beobachtungsstudien sind teils sehr unterschiedlich und fragwürdig. Deshalb ist es wichtig, die erzielten Resultate in randomisierten, placebokontrollierten Studien (RCT) unter Einschluss einer angemessenen Probandenzahl zu überprüfen. Nachfolgend werden 7 RCT zum Einsatz einer HT bei postmenopausalen Frauen näher betrachtet. Der Fokus ist auf die am häufigsten angewendeten HT-Präparate und deren Einfluss auf die Kognition gerichtet.
In der medizinischen Online-Datenbank PubMed wurde eine Literarturrecherche durchgeführt, wobei zwischen RCT zur Östrogenmonotherapie ["estrogen therapy“ (ET)] und Östrogen-Gestagen-Therapie ["estrogen-progestogen therapy" (EPT)] sowie zwischen Studien mit Probandinnen über bzw. unter 65 Jahren bei Studienstart unterschieden wurde. In den detektierten RCT wurde mit neuropsychologischen Tests der Einfluss einer HT auf die globale Kognition oder auf kognitive Teilgebiete untersucht.

\section{Ergebnisse}

Insgesamt erfüllten 7 RCT die Kriterien. Diese werden im Folgenden dargestellt. Die erste und größte RCT war die Women's Health Initiative Memory Study (WHIMS), eine Substudie der Women's Health Initiative (WHI), die als primären Endpunkt alle Arten der Demenz und als sekundäre Endpunkte leichte kognitive Beeinträchtigungen und die globalen kognitiven Funktionen bei gesunden postmenopausalen Frauen untersuchte [1]. Die Women's Health Initiative Study of Cognitive Aging (WHISCA) war ebenfalls eine Substudie der WHI, die verschiedene kognitive Domänen beleuchtete [8].

\section{Östrogentherapie bei über 65-Jährigen}

In - Tab. 1 sind 3 RCT zum Einfluss einer ET auf die globalen kognitiven Funktionen oder auf deren Teilgebiete bei Frauen in einem Alter über 65 Jahre bei Studienstart gezeigt. In der WHIMS [1] und
WHISCA [8] erfolgte die ET-Applikation mit konjugierten equinen Östrogenen (CEE) oral, in der Studie von Yaffe et al. [11] wurde dagegen Östradiol $\left(\mathrm{E}_{2}\right)$ transdermal appliziert. In der WHIMS war ein minimaler negativer Effekt der ET auf die globale Kognition zu beobachten. Dabei ist zu beachten, dass die globalen kognitiven Funktionen nur als sekundäre Endpunkte gemessen wurden. Als primärer Endpunkt wurden alle Arten der Demenz definiert. In den beiden anderen Studien konnte kein signifikanter negativer Effekt der ET ermittelt werden, aber auch kein positiver. Zusammenfassend legen die 3 Studien die Annahme nahe, dass Östrogene allein bei postmenopausalen Frauen über 65 Jahre keinen positiven Effekt auf die Kognition ausüben.

\section{Östrogen-Gestagen-Therapie bei über 65-Jährigen}

In - Tab. 2 sind 3 RCT zum Einfluss einer EPT auf die globalen kognitiven Funktionen oder auf deren Teilgebiete bei Frauen in einem Alter über 65 Jahre bei Studienstart aufgeführt. Alle Studien setzten CEE als Östrogenkomponente und Medroxyprogesteronacetat (MPA) als Gestagenkomponente ein. Unisono zeigen die 3 Studien einen negativen Effekt der EPT auf kognitive Fähigkeiten, wobei der negative Effekt in der WHISCA auf das verbale Gedächtnis beschränkt blieb und nur nach einer EPT-Langzeittherapie nachweisbar war - die EPT dauerte zwischen 1,1 und 5,6 Jahren, der Mittelwert lag bei 3,0 Jahren. In der Heart and Estrogen/progestin Replacement Study (HERS) wurde primär die präventive Wirkung einer HT auf Komplikationen 


\begin{tabular}{|c|c|c|c|c|c|c|c|}
\hline $\begin{array}{l}\text { Autor/ } \\
\text { Studie }\end{array}$ & $\begin{array}{l}\text { Teilneh- } \\
\text { mer (n) }\end{array}$ & $\begin{array}{l}\text { Kohorten- } \\
\text { charakteristika }\end{array}$ & $\begin{array}{l}\text { Hormonthe- } \\
\text { rapie/Dosis }\end{array}$ & Dauer & Outcome & Tests & Resultate \\
\hline $\begin{array}{l}\text { Women's } \\
\text { Health Ini- } \\
\text { tiative Me- } \\
\text { mory Study } \\
\text { (WHIMS; } \\
\text { [1]) }\end{array}$ & $\begin{array}{l}2947 \\
1464 \\
\text { (HT) } \\
1483 \\
\text { (Place- } \\
\text { bo) }\end{array}$ & $\begin{array}{l}\text { Gesunde } \\
\text { postmeno- } \\
\text { pausale Frauen } \\
\text { zwischen } 65 \\
\text { und } 79 \text { Jahren } \\
\text { (mittleres Alter: } \\
69 \text { Jahre) }\end{array}$ & $\begin{array}{l}\text { CEE: } \\
0,625 \mathrm{mg} / \mathrm{Tag} \\
\text { oral }\end{array}$ & $\begin{array}{l}\text { Durch- } \\
\text { schnitt: } \\
\text { 5,21 Jahre }\end{array}$ & $\begin{array}{l}\text { Primärer Endpunkt: alle Arten } \\
\text { der Demenz } \\
\text { Sekundärer Endpunkt: leichte } \\
\text { kognitive Beeinträchtigung } \\
\text { und globale kognitive Funk- } \\
\text { tionen }\end{array}$ & $\begin{array}{l}\text { 3MS, ausschließlich, } \\
\text { um die globalen ko- } \\
\text { gnitiven Funktionen } \\
\text { zu testen }\end{array}$ & $\begin{array}{l}\text { Minimal HT < } \\
\text { Placebo } \\
\text { Mittelwert des } \\
\text { 3MS war in der } \\
\text { HT-Gruppe um } \\
0,26 \text { Einheiten } \\
\text { niedriger als in } \\
\text { der Placebo- } \\
\text { gruppe }(p=0,04)\end{array}$ \\
\hline $\begin{array}{l}\text { Women's } \\
\text { Health Ini- } \\
\text { tiative Study } \\
\text { of Cogni- } \\
\text { tive Aging } \\
\text { (WHISCA; } \\
\text { [8]) }\end{array}$ & $\begin{array}{l}886 \\
434 \\
\text { (CEE) } \\
452 \text { (Pla- } \\
\text { cebo) }\end{array}$ & $\begin{array}{l}\text { Gesunde, hys- } \\
\text { terektomierte, } \\
\text { postmeno- } \\
\text { pausale Frauen } \\
\text { zwischen } 66 \\
\text { und } 84 \text { Jahren } \\
\text { (mittleres Alter: } \\
\text { 73,9 Jahre), oh- } \\
\text { ne Anzeichen } \\
\text { einer Demenz }\end{array}$ & $\begin{array}{l}\text { CEE: } \\
0,625 \mathrm{mg} / \mathrm{Tag} \\
\text { oral }\end{array}$ & $\begin{array}{l}\text { 1,1-5,6 Jah- } \\
\text { re (Mittel- } \\
\text { wert: } \\
3,0 \text { Jahre) }\end{array}$ & $\begin{array}{l}\text { Altersassoziierte Veränderun- } \\
\text { gen in den kognitiven Funk- } \\
\text { tionen über die Zeit, anhand } \\
\text { von verbalem und figuralem } \\
\text { Gedächtnis, Arbeitsgedächtnis, } \\
\text { Aufmerksamkeit, räumlichem } \\
\text { Denken, Geschwindigkeit der } \\
\text { mentalen Leistungen, exeku- } \\
\text { tiven Funktionen und motori- } \\
\text { scher Leistung }\end{array}$ & $\begin{array}{l}\text { PMA Vocabulary, Let- } \\
\text { ter Fluency, Semantic } \\
\text { Fluency, Benton Visu- } \\
\text { al Retention Test, Cali- } \\
\text { fornia Verbal Learning } \\
\text { Test, Digits Forward, } \\
\text { Digits Backward, Card } \\
\text { Rotations, Finger } \\
\text { Tapping (dominante } \\
\text { und nichtdominante } \\
\text { Hand) }\end{array}$ & $\begin{array}{l}\text { HT = Placebo } \\
\text { Keine signifikan- } \\
\text { ten Gruppendif- } \\
\text { ferenzen }\end{array}$ \\
\hline $\begin{array}{l}\text { Yaffe et al. } \\
\text { (2006; [11]) }\end{array}$ & $\begin{array}{l}417 \\
208(\mathrm{HT}) \\
209 \text { (Pla- } \\
\text { cebo) }\end{array}$ & $\begin{array}{l}\text { Frauen zwi- } \\
\text { schen } 60 \text { und } \\
80 \text { Jahren, post- } \\
\text { menopausal } \\
\text { (mindestens } \\
5 \text { Jahre nach } \\
\text { Menopause), } \\
\text { mit intaktem } \\
\text { Uterus }\end{array}$ & $\begin{array}{l}\text { Östradiol: } \\
0,014 \mathrm{mg} / \\
\text { Tag } \\
\text { (1 transder- } \\
\text { males Pflas- } \\
\text { ter/Woche) }\end{array}$ & 2 Jahre & $\begin{array}{l}\text { Veränderungen der kognitiven } \\
\text { Fähigkeiten über die Zeit }\end{array}$ & $\begin{array}{l}\text { 3MS, Logical Memory } \\
\text { (,„immediate and } \\
\text { delayed"), Brief Visuo- } \\
\text { spatial Memory Test, } \\
\text { Word List Memory, } \\
\text { Trail Making Test } \\
\text { (Form B), Modified } \\
\text { Boston Naming Test, } \\
\text { Verbal Fluency }\end{array}$ & $\begin{array}{l}\text { HT = Placebo } \\
\text { Keine signifikan- } \\
\text { ten Gruppendif- } \\
\text { ferenzen }\end{array}$ \\
\hline
\end{tabular}

der koronaren Herzkrankheit (Myokardinfarkt und plötzlicher Herztod) eruiert; eingeschlossen waren postmenopausale Frauen mit nachgewiesener koronarer Herzkrankheit [3]. In einer Nebenstudie wurde die Wirkung der HT auf die globalen kognitiven Funktionen untersucht. Dabei beschränkte sich der negative Effekt auf den verbalen Fluss und das Wörterlistengedächtnis. Wie weit die klinisch relevante koronare Herzkrankheit der Probandinnen in der HERS die kognitiven Fähigkeiten beeinflusste, ist unklar. Aus den Ergebnissen der 3 Studien lässt sich die Hypothese ableiten, dass CEE in Kombination mit MPA einen negativen Effekt auf die Kognition von postmenopausalen Frauen über 65 Jahre haben.

\section{Östrogen-Gestagen-Therapie bei unter 65-Jährigen}

In - Tab. 3 sind 2 RCT mit EPT bei postmenopausalen Frauen unter 65 Jahren bei Studienstart gezeigt. Die Hormonexposi- tion erfolgte also mit einer kleineren zeitlichen Distanz zur natürlichen Menopause als in den Studien aus 0 Tab. 1 und 2. Auch die Dauer der Exposition ist kürzer als in den o. g. Studien. In der Studie von Maki et al. [5] litten die Probandinnen an mindestens einer subjektiven kognitiven Beschwerde. Neben Abweichungen der Gedächtnisleistung und der Aufmerksamkeit von den Basiswerten wurde auch die Symptomverbesserung der subjektiven kognitiven Beschwerden erfasst. Ein signifikanter Unterschied zwischen den beiden Studienarmen ließ sich nicht ermitteln. Allerdings zeigte sich in der HT-Gruppe (CEE plus MPA) ein geringer negativer Effekt auf das verbale Gedächtnis: In den CaliforniaVerbal-Learning-Untertests „Short-delay“ und "Long-delay Free Recall“ kam es in der HT-Gruppe zu Leistungseinbußen [5]. Weil aber die notwendige Probandenzahl nicht erreicht wurde, hat die Studie nicht genügend Aussagekraft, um etwaige Gruppenunterschiede eindeutig zu detektieren.
In der Studie von Sherwin et al. [10] gab es neben der Placebogruppe und der Hormongruppe mit CEE und MPA noch eine weitere Hormongruppe mit CEE und mikronisiertem Progesteron. In der CEE+MPA-Gruppe konnte kein Unterschied zur Placebogruppe detektiert werden. In der Gruppe mit CEE und Progesteron ließen sich dagegen interessanterweise ein positiver und ein negativer Effekt auf 2 verschiedene Teilgebiete der Kognition nachweisen: ein negativer Effekt auf das verzögerte verbale Gedächtnis mit Sitz im Hippocampus und ein positiver Effekt auf das Arbeitsgedächtnis mit Sitz im präfrontalen Kortex [10]. Der negative Effekt zeigte sich in einer signifikanten Punktedifferenz zwischen dem Test vor der Hormonexposition und dem Test nach Behandlung. Da diese beiden kognitiven Funktionen mit unterschiedlichen Hirnstrukturen assoziiert sind, ist davon auszugehen, dass Progesteron in den verschiedenen Hirnstrukturen unterschiedliche Wirkungen vermittelt. 
Auch in diesen beiden Studien konnte kein signifikanter Gruppenunterschied zwischen der Placebo- und der EPT-Gruppe detektiert werden. Dies steht im Gegensatz zum detektierbaren negativen Effekt von CEE und MPA auf die Kognition bei Probandinnen über 65 Jahre (• Tab. 2). Zu einem ähnlichen Fazit kommt die Kronos Early Estrogen Prevention Study (KEEPS), deren Ergebnisse zur Kognition 2013 auf der Jahrestagung der North American Menopause Society präsentiert wurden.

\section{Östrogentherapie bei unter 65-Jährigen}

In - Tab. 4 ist eine RCT mit ET bei Frauen unter 65 Jahren bei Studienstart aufgeführt. Die Studie beinhaltet 3 Studienarme mit $E_{2}$, Testosteron oder Placebo [4]. Untersucht wurde der Einfluss der exogenen Hormonexposition auf spezifische kognitive Fähigkeiten, wie das verbale Gedächtnis, den verbalen Fluss und das räumliche Denkvermögen. Die neuropsychologischen Tests erfolgten nach 4-wöchiger Hormonsubstitution. Dabei konnten keine signifikanten Unterschiede in den kognitiven Leistungen zwischen den Hormongruppen und der Placebogruppe nachgewiesen werden. Die Studie widerlegt damit den positiven Effekt einer HT auf die Kognition auch bei jüngeren postmenopausalen Frauen.

\section{Diskussion}

Wie aus - Tab. 1, 2, 3 und 4 ersichtlich ist, konnte nur eine der 7 näher betrachteten RCT einen signifikanten positiven Effekt der postmenopausalen HT auf die Kognition nachweisen - und das nur in einer Untergruppe des HT-Studienarms. Dies ist ein überraschendes Ergebnis, da in verschiedenen Beobachtungsstudien ein positiver Effekt ermittelt werden konnte. Für das Fehlen eines positiven Effekts der HT in RCT werden folgende Erklärungsansätze diskutiert: Die HT wird hauptsächlich zur Behandlung von vasomotorischen Störungen eingesetzt. Sie sind typisch für die Peri- und Postmenopause. Ein Großteil der in die Beobachtungsstudien eingeschlossenen Frauen litt also an vasomotorischen Störungen, die u. a. auch für die weitverbreiteten Schlafstörungen in der Postme-

Gynäkologische Endokrinologie 2015 · 13:64-68 DOI 10.1007/s10304-014-0660-0

(c) Springer-Verlag Berlin Heidelberg 2014

\section{Widmer $\cdot$ P. Stute \\ Postmenopausale Hormontherapie und Kognition}

\section{Zusammenfassung}

Hintergrund. Etwa $60 \%$ der Frauen in den Wechseljahren klagen über Gedächtniseinbußen. In Beobachtungsstudien konnte ein positiver Effekt der postmenopausalen Hormontherapie (HT) auf die Kognition nachgewiesen werden.

Fragestellung. Kann der positive Effekt der HT auf die Kognition in randomisierten, placebokontrollierten Studien (RCT) reproduziert werden?

Material und Methode. Es wurde eine systematische Literaturrecherche durchgeführt. Unterschieden wurde zwischen RCT zur Östrogenmonotherapie und Östrogen-Gestagen-Therapie sowie zwischen Probandinnen über und unter 65 Jahren bei Studienstart. Ergebnisse. Die detektierten RCT zeigen mehrheitlich keinen positiven oder negati- ven Einfluss einer HT auf die Kognition. Bei Frauen über 65 Jahre hat die kombinierte HT mit konjugierten equinen Östrogenen und Medroxyprogesteronacetat einen negativen Einfluss auf die Kognition, bei Frauen unter 65 Jahren jedoch nicht.

Schlussfolgerung. Der in Beobachtungsstudien gezeigte positive Effekt einer HT auf die Kognition lässt sich nicht ohne weiteres in RCT reproduzieren. Es stellt sich weiterhin die Frage nach einem kognitiven „window of opportunity".

\section{Schlüsselwörter}

Gedächtnisstörungen · Randomisierte, kontrollierte Studien · Östrogene . Gestagene - Einflussfaktor Alter

\section{Postmenopausal hormone therapy and cognition}

\section{Abstract}

Background. Approximately $60 \%$ of women undergoing menopause complain about loss of memory. A positive effect of postmenopausal hormone therapy $(\mathrm{HT})$ on cognition was found in observational studies. Question. Can the positive effect of postmenopausal HT on cognition be reproduced in randomized, placebo-controlled trials (RCT)?

Material and methods. A systematic literature search was carried out. A distinction was made between RCTs for estrogen monotherapy (ET) and estrogen-progestogen therapy (EPT) as well as between subjects above and below 65 years of age at study entry.

Results. The majority of the RCTs demonstrated neither a positive nor a negative ef- fect of postmenopausal HT on cognition. In women above the age of 65 years treated with conjugated equine estrogens and medroxyprogesterone acetate, a negative impact of HT on cognition was shown but not in women below 65 years old.

Conclusion. The positive effect of postmenopausal HT on cognition found in observational studies was not easily reproducible in RCTs. Furthermore, it remains to be proven if there is a cognitive window of opportunity.

\section{Keywords}

Memory disorders $\cdot$ Randomized controlled trials · Estrogens · Progestogens · Age factors nopause verantwortlich sind. Auch die mit der Menopause auftretenden subjektiven kognitiven Beschwerden können durch die Schlafstörungen und vasomotorischen Störungen erklärt werden. Dieser Sachverhalt führt zur Hypothese, dass eine Linderung der vasomotorischen Beschwerden der direkte positive Effekt auf die Kognition sein könnte, welchen man der HT zugeschrieben hatte. Aus den 7 hier diskutierten Studien ist nicht ersichtlich, ob die Probandinnen an starken vasomotorischen Störungen litten.

\section{D) Die Ergebnisse sind möglicherweise durch einen gesünderen Lebensstil in der HT-Gruppe verzerrt}

Ein weiterer Diskussionspunkt ist die Verzerrung der Studienergebnisse durch gesunde Therapieanwender („healthy user bias"), die eventuell mit den Beobachtungsstudien assoziiert ist. Die Analysie der Fall-Kontroll-Studien ergab, dass die Frauen mit HT im Durchschnitt einen höheren Bildungsgrad und oft einen gesünderen 
Tab. 2 Randomisierte, placebokontrollierte Studien mit konjugierten equinen Östrogenen plus Medroxyprogesteronacetat bei

postmenopausalen Frauen über 65 Jahre

\begin{tabular}{|c|c|c|c|c|c|c|c|}
\hline $\begin{array}{l}\text { Autor/ } \\
\text { Studie }\end{array}$ & $\begin{array}{l}\text { Teilnehmer } \\
\text { (n) }\end{array}$ & $\begin{array}{l}\text { Kohorten- } \\
\text { charakteristika }\end{array}$ & Dosis & Dauer & Outcome & Tests & Resultate \\
\hline $\begin{array}{l}\text { Women's } \\
\text { Health Initia- } \\
\text { tive Memory } \\
\text { Study } \\
\text { (WHIMS; [1]) }\end{array}$ & 4532 & $\begin{array}{l}\text { Gesunde post- } \\
\text { menopausale } \\
\text { Frauen zwischen } \\
65 \text { und } 79 \text { Jah- } \\
\text { ren (mittleres } \\
\text { Alter: } 69 \text { Jahre) }\end{array}$ & $\begin{array}{l}\text { CEE: } \\
0,625 \mathrm{mg} / \\
\text { Tag } \\
\text { MPA: } \\
2,5 \mathrm{mg} / \\
\text { Tag }\end{array}$ & $\begin{array}{l}\text { Durch- } \\
\text { schnitt: } \\
4,05 \text { Jahre }\end{array}$ & $\begin{array}{l}\text { Primärer Endpunkt: alle } \\
\text { Arten der Demenz } \\
\text { Sekundärer Endpunkt: } \\
\text { leichte kognitive Beein- } \\
\text { trächtigung und globale } \\
\text { kognitive Funktionen }\end{array}$ & $\begin{array}{l}\text { 3MS, ausschließlich, } \\
\text { um die globalen kog- } \\
\text { nitiven Funktionen zu } \\
\text { testen }\end{array}$ & $\begin{array}{l}\text { HT< Placebo } \\
\text { Klinisch relevanter } \\
\text { Rückgang in den } \\
\text { 3MS-Leistungen: } \\
6,7 \% \text { in der HT- } \\
\text { Gruppe, } 4,8 \% \text { in } \\
\text { der Placebogruppe } \\
\text { ( }=0,008)\end{array}$ \\
\hline $\begin{array}{l}\text { Women's } \\
\text { Health Ini- } \\
\text { tiative Study } \\
\text { of Cogni- } \\
\text { tive Aging } \\
\text { (WHISCA; } \\
\text { [7]) }\end{array}$ & 1416 & $\begin{array}{l}\text { Gesunde post- } \\
\text { menopausale } \\
\text { Frauen zwischen } \\
66 \text { und } 84 \text { Jah- } \\
\text { ren (mittleres Al- } \\
\text { ter: } 73,9 \text { Jahre), } \\
\text { ohne Anzeichen } \\
\text { einer Demenz }\end{array}$ & $\begin{array}{l}\text { CEE: } \\
0,625 \mathrm{mg} / \\
\text { Tag } \\
\text { MPA: } \\
\text { 2,5 mg/ } \\
\text { Tag }\end{array}$ & $\begin{array}{l}\text { 1,1-5,6 Jah- } \\
\text { re (Mit- } \\
\text { telwert: } \\
3,0 \text { Jahre) }\end{array}$ & $\begin{array}{l}\text { Altersassoziierte Ver- } \\
\text { änderungen in den kog- } \\
\text { nitiven Funktionen über } \\
\text { die Zeit, anhand von } \\
\text { verbalem und figuralem } \\
\text { Gedächtnis, Arbeits- } \\
\text { gedächtnis, Aufmerk- } \\
\text { samkeit, räumlichem } \\
\text { Denken, Geschwin- } \\
\text { digkeit der mentalen } \\
\text { Leistungen, exekutiven } \\
\text { Funktionen und motori- } \\
\text { scher Leistung }\end{array}$ & $\begin{array}{l}\text { PMA Vocabulary, Let- } \\
\text { ter Fluency, Semantic } \\
\text { Fluency, Benton Visual } \\
\text { Retention Test, CVLT, } \\
\text { Digits Forward, Digits } \\
\text { Backward, Card Rota- } \\
\text { tions, Finger Tapping } \\
\text { (dominante und nicht- } \\
\text { dominante Hand) }\end{array}$ & $\begin{array}{l}\mathrm{HT}<\text { Placebo } \\
\text { Nur negativer Effekt } \\
\text { auf verbales Ge- } \\
\text { dächtnis ( } \mathrm{p}=0,01 \text { ) } \\
\text { und nur ersichtlich } \\
\text { nach Langzeitbe- } \\
\text { handlung }\end{array}$ \\
\hline $\begin{array}{l}\text { Heart and } \\
\text { Estrogen/ } \\
\text { progestin } \\
\text { Replace- } \\
\text { ment Study } \\
\text { (HERS), } \\
\text { Grady et al. } \\
(2002 ;[3])\end{array}$ & $\begin{array}{l}1063 \\
517 \text { (HT- } \\
\text { Gruppe) } \\
546 \text { (Place- } \\
\text { bogruppe) }\end{array}$ & $\begin{array}{l}\text { Postmeno- } \\
\text { pausale Frauen } \\
\text { unter } 80 \text { Jahren } \\
\text { (Mittelwert: } \\
71 \pm 6 \text { Jahre), mit } \\
\text { intaktem Uterus } \\
\text { und nachgewie- } \\
\text { sener koronarer } \\
\text { Herzkrankheit }\end{array}$ & $\begin{array}{l}\text { CEE: } \\
0,625 \mathrm{mg} / \\
\text { Tag } \\
\text { MPA: } \\
2,5 \mathrm{mg} / \\
\text { Tag }\end{array}$ & $\begin{array}{l}\text { Mittelwert: } \\
4,2 \pm 0,4 \text { Jah- } \\
\text { re }\end{array}$ & $\begin{array}{l}\text { Globale kognitive Fähig- } \\
\text { keiten }\end{array}$ & $\begin{array}{l}\text { 3MS, Verbal Fluency, } \\
\text { Boston Naming, Word } \\
\text { List Memory, Word List } \\
\text { Recall, Trail Making } \\
\text { Test (Form B) }\end{array}$ & $\begin{array}{l}\text { HT }<\text { Placebo } \\
\text { In Verbal Fluency } \\
(p=0,02) \text { und } \\
\text { Word List Memory } \\
(p=0,06)\end{array}$ \\
\hline
\end{tabular}

Lebensstil aufwiesen. Die Möglichkeit der Verzerrung durch einen gesünderen Lebensstil in der HT-Gruppe darf daher nicht außer Acht gelassen werden.

Trotz einschneidender Ergebnisse der WHI besteht immer noch ein beachtlicher HT-Bedarf in der Peri- und Postmenopause. In diesen Phasen wird sie hauptsächlich zur Linderung vasomotorischer Beschwerden eingesetzt. Deshalb stellt sich nicht nur die Frage, ob eine HT einen positiven Effekt auf die Kognition hat, sondern vielmehr auch, wie man die in RCT nachgewiesenen negativen Wirkungen auf die Kognition verhindern oder minimieren kann.

In der Studie von Sherwin et al. [10] konnte kein negativer Effekt der Einnahme von CEE und MPA auf die Kognition nachgewiesen werden. Dies steht im Gegensatz zu den Ergebnissen der WHIMS und HERS $[1,3]$. Ein wesentlicher Unterschied zwischen den Studien war das Alter der Probandinnen. In der WHIMS und HERS waren die Probandinnen wesentlich älter, damit lag zwischen Menopause und HT-Beginn auch eine längere Zeitspanne. Eventuell könnte ein rascher Beginn der HT nach der Menopause den negativen Effekt der HT auf die Kognition verhindern oder minimieren. Dies gilt es in weiteren klinischen Studien zu überprüfen.

\section{Fazit für die Praxis}

\section{- Subjektive Gedächtnisstörungen in der Postmenopause sind häufig.}

- Der positive Effekt der HT auf die Kognition, wie er aus Beobachtungsstudien bekannt ist, ist in RCT mit Östrogenen und Gestagenen nicht reproduzierbar.

- Die postmenopausale HT mit Östrogenen in Kombination mit Gestagenen hat bei postmenopausalen Frauen über 65 Jahre einen negativen Effekt auf die Kognition.

- Die HT mit weiblichen Sexualhormonen ist die wirksamste Therapie zur
Linderung der vasomotorischen Störungen in den Wechseljahren. Sehr wahrscheinlich hat sie auch einen positiven Effekt auf die subjektiven kognitiven Störungen der Postmenopause.

- Aktuell gelten kognitive Beschwerden in der Postmenopause nicht als Indikation für eine HT. Es bleibt abzuwarten, ob die Hypothese des kognitiven „,window of opportunity" bestätigt werden kann.

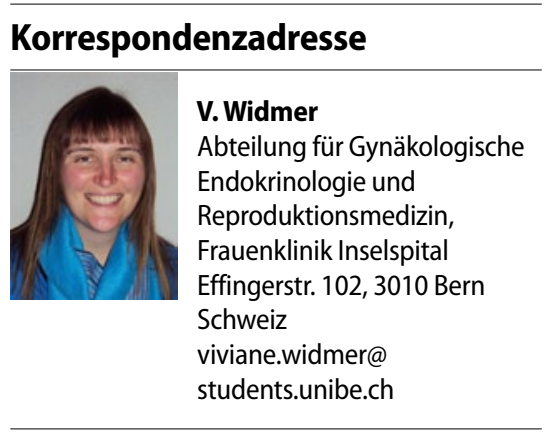


Tab. 3 Randomisierte, placebokontrollierte Studien mit konjugierten equinen Östrogenen plus Gestagene bei postmenopausalen Frauen unter 65 Jahren

\begin{tabular}{|c|c|c|c|c|c|c|c|}
\hline $\begin{array}{l}\text { Autor/ } \\
\text { Studie }\end{array}$ & $\begin{array}{l}\text { Teilnehmer } \\
\text { (n) }\end{array}$ & $\begin{array}{l}\text { Kohorten- } \\
\text { charakteristika }\end{array}$ & $\begin{array}{l}\text { Hormonthe- } \\
\text { rapie/Dosis }\end{array}$ & Dauer & Outcome & Tests & Resultate \\
\hline $\begin{array}{l}\text { Maki et al. } \\
(2007 ;[5])\end{array}$ & 180 & $\begin{array}{l}\text { Postmenopausale } \\
\text { Frauen zwischen } \\
45 \text { und } 55 \text { Jahren, } \\
\text { deren Menopau- } \\
\text { se nicht länger als } \\
3 \text { Jahre zurücklag, } \\
\text { mit mindestens } \\
\text { einer subjektiven } \\
\text { kognitiven Be- } \\
\text { schwerde }\end{array}$ & $\begin{array}{l}\text { CEE: } \\
0,625 \mathrm{mg} / \mathrm{Tag} \\
\text { MPA: } 2,5 \mathrm{mg} / \\
\text { Tag }\end{array}$ & 4 Monate & $\begin{array}{l}\text { Abweichungen } \\
\text { der Gedächt- } \\
\text { nisleistung, der } \\
\text { Aufmerksamkeit } \\
\text { und der subjek- } \\
\text { tiven kognitiven } \\
\text { Leistung von } \\
\text { den gemessenen } \\
\text { Grundwerten }\end{array}$ & $\begin{array}{l}\text { California Verbal } \\
\text { Learning Test (CVLT), } \\
\text { Memory Function } \\
\text { Questionnaire (MFQ), } \\
\text { Brief Test of Atten- } \\
\text { tion (BTA) }\end{array}$ & $\begin{array}{l}\mathrm{HT}=\text { Placebo } \\
+ \text { geringer negativer Ef- } \\
\text { fekt der Hormontherapie } \\
\text { auf das verbale Gedächt- } \\
\text { nis }(p<0,10)\end{array}$ \\
\hline $\begin{array}{l}\text { Sherwin } \\
\text { et al. (2011; } \\
[10])\end{array}$ & $\begin{array}{l}24 \\
7 \text { (CEE+Pla- } \\
\text { cebo) } \\
8 \text { (CEE+MP) } \\
9 \text { (CEE+MPA) }\end{array}$ & $\begin{array}{l}\text { Frauen mit natür- } \\
\text { licher Menopause } \\
\text { und intaktem } \\
\text { Uterus, zwischen } \\
50 \text { und } 55 \text { Jahren, } \\
\text { ohne voran- } \\
\text { gegangene Hor- } \\
\text { montherapie }\end{array}$ & $\begin{array}{l}\text { CEE: } \\
\text { 0,625 mg/Tag } \\
\text { für } 28 \text { Tage } \\
\text { (=Therapie- } \\
\text { zyklus) } \\
\text { MP: } 200 \mathrm{mg} / \\
\text { Tag, Tag 1-14/ } \\
\text { Therapie- } \\
\text { zyklus } \\
\text { MPA: } 10 \mathrm{mg} / \\
\text { Tag, Tag 1-14/ } \\
\text { Therapie- } \\
\text { zyklus } \\
\text { Placebo: } \\
\text { Tag 1-14/ } \\
\text { Therapie- } \\
\text { zyklus }\end{array}$ & 3 Monate & $\begin{array}{l}\text { Veränderungen } \\
\text { verschiedener } \\
\text { kognitiver Funk- } \\
\text { tionen über die } \\
\text { Zeit (verbales Ge- } \\
\text { dächtnis, Arbeits- } \\
\text { gedächtnis, räum- } \\
\text { liches Vorstel- } \\
\text { lungsvermögen, } \\
\text { "visual-spacial } \\
\text { sequencing“) }\end{array}$ & $\begin{array}{l}\text { Logical Memory Test } \\
\text { („immediate and } \\
\text { delayed“), Paired As- } \\
\text { sociates Test („,imme- } \\
\text { diate and delayed“), } \\
\text { Digit Span Forward, } \\
\text { Digit Span Backward, } \\
\text { Mental Rotations } \\
\text { Test, Trail Making } \\
\text { Test (Form B) }\end{array}$ & $\begin{array}{l}\mathrm{CEE}+\mathrm{MPA}=\text { Placebo } \\
\mathrm{CEE}+\mathrm{MP}<\text { Placebo (im } \\
\text { verzögerten verbalen } \\
\text { Gedächtnis, } \mathrm{p}=0,022 \text { ) } \\
\mathrm{CEE}+\mathrm{MP}>\text { Placebo (im } \\
\text { Arbeitsgedächtnis, } \\
\mathrm{p}=0,008 \text { ) }\end{array}$ \\
\hline
\end{tabular}

Tab. 4 Randomisierte, placebokontrollierte Studie mit Östradiol (keine Gestagene) bei postmenopausalen Frauen unter 65 Jahren

\begin{tabular}{|c|c|c|c|c|c|c|c|}
\hline Autor & $\begin{array}{l}\text { Teilnehmer } \\
\text { (n) }\end{array}$ & Kohortencharakteristika & Dosis & Dauer & Outcome & Tests & Resultate \\
\hline $\begin{array}{l}\text { Kocoska-Maras } \\
\text { et al. }(2011 ;[4])\end{array}$ & $\begin{array}{l}203 \\
66\left(E_{2}\right) \\
67(T) \\
67 \text { (Placebo) }\end{array}$ & $\begin{array}{l}\text { Frauen mit natürlicher Me- } \\
\text { nopause zwischen } 50 \text { und } \\
65 \text { Jahren, ohne Hormon- } \\
\text { therapie in den vorange- } \\
\text { gangenen } 3 \text { Monaten }\end{array}$ & $\begin{array}{l}\mathrm{E}_{2}: 2 \mathrm{mg} / \mathrm{Tag} \\
\mathrm{T}: 40 \mathrm{mg} / \mathrm{Tag}\end{array}$ & 4Wochen & $\begin{array}{l}\text { Verbal Fluency, } \\
\text { Verbal Me- } \\
\text { mory, Spatial } \\
\text { Ability }\end{array}$ & $\begin{array}{l}\text { Letter Fluency } \\
\text { Test, Word List } \\
\text { Memory, Men- } \\
\text { tal Rotation }\end{array}$ & $\mathrm{HT}=$ Placebo \\
\hline
\end{tabular}

\section{Einhaltung ethischer Richtlinien}

Interessenkonflikt. V. Widmer und P. Stute geben an, dass kein Interessenkonflikt besteht.

\section{Literatur}

1. Coker LH, Espeland MA, Rapp SR et al (2010) Postmenopausal hormone therapy and cognitive outcomes: the Women's Health Initiative Memory Study (WHIMS). J Steroid Biochem Mol Biol 118(45):304-310

2. Fischer B, Gleason C, Asthana S (2014) Effects of hormone therapy on cognition and mood. Fertil Steril 101(4):898-904

3. Grady D, Yaffe K, Kristof M et al (2002) Effect of postmenopausal hormone therapy on cognitive function: the Heart and Estrogen/progestin Replacement Study. Am J Med 113(7):543-548
4. Kocoska-Maras L, Zethraeus N, Rådestad AF et al (2011) A randomized trial of the effect of testosterone and estrogen on verbal fluency, verbal memory, and spatial ability in healthy postmenopausal women. Fertil Steril 95(1):152-157

5. Maki PM, Gast MJ, Vieweg AJ et al (2007) Hormone therapy in menopausal women with cognitive complaints: a randomized, double-blind trial. Neurology 69(13):1322-1330

6. Maki PM, Sundermann E (2009) Hormone therapy and cognitive function. Hum Reprod Update 15(6):667-681

7. Bergen M von (2013) Das Gedächtnis in der Perimenopause - Die kognitiven Veränderungen während der Wechseljahre. Universität Bern, Bern

8. Resnick SM, Espeland MA, An Y et al (2009) Effects of conjugated equine estrogens on cognition and affect in postmenopausal women with prior hysterectomy. J Clin Endocrinol Metab 94(11):41524161
9. Ryan J, Stanczyk FZ, Dennerstein L et al (2012) Hormone levels and cognitive function in postmenopausal midlife women. Neurobiol Aging 33(7):1138-1147

10. Sherwin BB, Grigorova M (2011) Differential effects of estrogen and micronized progesterone or medroxyprogesterone acetate on cognition in postmenopausal women. Fertil Steril 96(2):399-403

11. Yaffe $K$, Vittinghoff $E$, Ensrud KE et al (2006) Effects of ultra-low-dose transdermal estradiol on cognition and health-related quality of life. Arch Neurol 63(7):945-950 